\title{
Sustainable Energy Consumption Monitoring in Residential Settings
}

\author{
Akshay Uttama Nambi S. N. ${ }^{1}$, Thanasis G. Papaioannou ${ }^{1}$, Dipanjan Chakraborty ${ }^{2}$ and Karl Aberer ${ }^{1}$ \\ ${ }^{1}$ School of Computer and Communication Sciences \\ Ecole Polytechnique Fédérale de Lausanne \\ CH-1015 Lausanne, Switzerland \\ 2 IBM Research - India Lab, New Delhi, India \\ akshay.uttama@gmail.com, \{thanasis.papaioannou, karl.aberer\}@epfl.ch, cdipanjan@in.ibm.com
}

\begin{abstract}
The continuous growth of energy needs and the fact that unpredictable energy demand is mostly served by unsustainable (i.e. fossil-fuel) power generators have given rise to the development of Demand Response (DR) mechanisms for flattening energy demand. Building effective DR mechanisms and user awareness on power consumption can significantly benefit from fine-grained monitoring of user consumption at the appliance level. However, installing and maintaining such a monitoring infrastructure in residential settings can be quite expensive. In this paper, we study the problem of fine-grained appliance power-consumption monitoring based on one houselevel meter and few plug-level meters. We explore the trade-off between monitoring accuracy and cost, and exhaustively find the minimum subset of plug-level meters that maximize accuracy. As exhaustive search is time- and resource-consuming, we define a heuristic approach that finds the optimal set of plug-level meters without utilizing any other sets of plug-level meters. Based on experiments with real data, we found that few plug-level meters when appropriately placed - can very accurately disaggregate the total real power consumption of a residential setting and verified the effectiveness of our heuristic approach.
\end{abstract}

Keywords-Energy disaggregation; Hidden Markov Models; FHMM; NILM; plug-level meter

\section{INTRODUCTION}

Demand Response (DR) mechanisms aim to provide pricebased or other incentives to the users to shift their energy loads and flatten their daily energy consumption. The effectiveness of DR mechanisms in turn depends on how much finegrained information is available about users. For instance, if we knew appliance-level usage of residential users, it can be used for selection of the pricing or incentive mechanism that maximizes the effectiveness of DR policies. According to [1], there are 4 different consumption types of appliances, namely i) Permanent consumer devices, (ii) On-off appliances, (iii) Multistate appliances, (iv) Continuously variable consumer devices, where price-based incentive mechanisms can be effective only for types i) and ii). Based on appliance-level consumption profiling, the DR mechanism designer knows the highest energy consuming appliances (subject to power consumption reduction) and time of appliance usage (for deriving load shiftability during peak hours). Moreover, accurate consumption profiling is essential for the calculation of the consumption baseline, based on which the effectiveness of any DR mechanism is measured. Fine-grained monitoring and modeling energy consumption in houses can be achieved by a) an intrusive method, where each appliance in the house is monitored separately, b) a non-intrusive load monitoring (NILM) [2], where a single residential energy meter is used for estimating the individual appliance usage information based on appliance consumption signatures (i.e. consumption states, e.g. on/stand-by/off, real power, reactive power, voltage and current waveforms, etc.), historical data or user annotations. While NILM has been investigated extensively [1], the accuracy of achievable disaggregation is limited, while the generalization of the models trained from certain houses for the energy consumption disaggregation of others is ineffective. On the other hand, intrusive methods involve installing plug-level meters for each appliance in the house and have high investment and maintenance cost. This paper explores an approach to minimize the intrusion, while maximizing the disaggregation performance. It is based on the intuition that a few selected appliances, if monitored can help to disaggregate the total load quite efficiently. In order to achieve sustainability of our monitoring approach, we try to minimize intrusion and associated capital and operational expenses of plug-level monitoring by selectively installing the minimum number of plug-level meters per house, so as to achieve very high disaggregation accuracy. The optimal locations of plug-level meters are found based on exhaustive search. For avoiding time-consumption and resource-expenses, we propose a heuristic approach that is experimentally proved to always find the optimal set of plug-level meters. Based on experiments with real power consumption data, we find that our approach can achieve very accurate load disaggregation with few plug-level meters installed in residential settings.

The remainder of this paper is organized as follows: In Section II, we overview the related work. In Section III, we set the goals of our monitoring approach. In Section IV, we describe the model for appliance-level energy consumption in houses. In Section V, we describe our approach for sustainable appliance monitoring based on exhaustive search and based on a heuristic. In Section VI, we experimentally verify the high accuracy of our monitoring approach and the effectiveness of the proposed heuristic. Finally, in Section VII, we conclude our work. 


\section{RELATED WORK}

Several research efforts are been carried out in order to design smart home environment and energy management systems for increasing user power consumption awareness [3]. In these systems, individual appliances in the house form a home area network. Also, various research efforts are in process to develop low-cost, reliable sensors and energy meters [4]. NILM techniques [1] employ machine learning methods to develop models for disaggregating residential power consumption at the appliance level. Hart et al. [2] describes a NILM technique to detect the appliances from the aggregated signal using "edges", in order to determine whether a appliance was turned on or off. Other works look at the steady-state power consumption for learning appliance signatures. In [5], transient noise of an electrical signal occurred during turning on or off of an appliance is used to determine the appliances and their signatures from the aggregated signal. In [6], plug-level energy readings are used to disaggregate the energy consumption of each appliance from the aggregated signal. In [7], they propose a novel algorithm using sparse coding dictionaries for disaggregating energy. All these works have the burden of installing and maintaining energy meters for each appliance.

Also, pattern recognition approaches for disaggregating the combined energy signal is employed in [8]. In [9], the authors try to disaggregate the energy information using sensors like acoustic, magnetic and light placed near to the appliances. Several features of the electrical signal from the energy meter to disaggregate energy information are employed in [10]. On the other hand, in [11], the authors propose to disaggregate the energy consumption from a single smart meter by using some prior knowledge of appliances with the help of a domain expert. An unsupervised technique to disaggregate energy consumption using factorial hidden markov models (FHMMs) is proposed in [12]. In [13], a Markovian model is proposed for home energy consumption, storage sizing and transformer sizing in the distributed network. In [14], a Communication and Energy Care Unit (CECU) models the energy consumption of a house based on pre-stored appliance signatures to determine the appliances from the aggregated signal. However, these techniques need either users to manually identify the appliances or assume full knowledge on the appliances in the house and their consumption signatures.

To summarize, none of the prior approaches have investigated sustainable approaches for reducing metering instrumentation, while achieving maximum disaggregation effectiveness. In this paper, we propose a sustainable approach to monitor energy usage of appliances using the aggregated signal: we select the minimum subset of appliances that need to be individually monitored, in order to achieve high disaggregation accuracy based on a heuristic algorithm.

\section{THE OBJECTIVES}

In this work, our objective is to develop a methodology for sustainable energy monitoring in houses. We try to maximize the accuracy of appliance-level energy monitoring using aggregated power signal, while minimizing the number of additional plug-level meters required to achieve this accuracy. Our optimization problem OPT can be summarized as follows:

OPT : $\max P:$ maximize energy monitoring accuracy $\min N$ : minimize the number of plug-level meters

where $P$ represents the accuracy of disaggregation achieved and $N$ indicates the number of sensors required for achieving this accuracy.

\section{Modeling Energy Consumption of Houses}

In this section, we describe the probabilistic models that we employ to model the total per-house energy consumption and that of individual appliances. We employ Hidden Markov Models (HMM) to model the time series of aggregated power readings of a house. HMMs has the ability to capture complex aggregate signals via a compact structure, such models have been used in areas such as speech recognition, audio separation and computational biology.

\section{A. Hidden Markov Models (HMMs)}

A discrete-time HMM can be viewed as a Markov model with hidden states characterized by a probability distribution function. A HMM can be described as follows: $N$ - Number

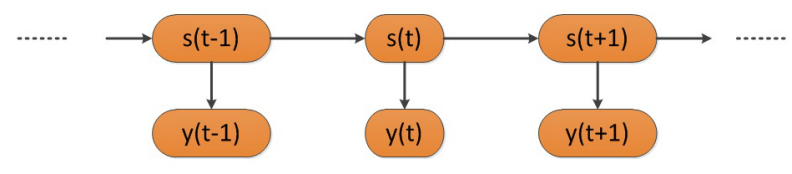

Fig. 1. Simple Hidden Markov Model (HMM).

of hidden states (e.g. on, stand-by, power-save, off etc.) of an appliance. Each state $s$ can be reached by any other state, $s=\left\{s_{1}, s_{2}, \ldots, s_{N}\right\} . M-$ Number of distinct symbols (i.e. energy consumption) observed in each state. $A$ - Transition matrix representing the probability of moving from state $s_{i}$ to $s_{j} . B$ - Emission matrix representing the probability of emission of a symbol in the current state. $\pi$ - Initial state probability distribution.

A graphical representation of HMM model is as shown in Fig. 1. Given a sequential consumption data $y=$ $\left\{y_{1}, y_{2}, y_{3}, \ldots, y_{T}\right\}$, each $y_{t}$ is generated by hidden state $s_{t}$.

\section{B. Factorial Hidden Markov Models (FHMMs)}

An extension of simple HMM is a Factorial HMM [15] to model multiple independent hidden state sequences, as shown in the Fig 2. Let us consider the sequential data $Y=\left\{y_{1}, y_{2}, y_{3}, \ldots, y_{T}\right\}$ representing the aggregated power signal of a house. Then, $s=\left\{s^{(1)}, s^{(2)}, \ldots, s^{(M)}\right\}$ represents the underlying state sequences, where $s^{i}=\left\{s_{1}^{(i)}, s_{2}^{(i)}, \ldots, s_{T}^{(i)}\right\}$ is the set of hidden states of chain $i$ in the FHMM model. In this work, we consider the states of the appliances in the house as the hidden states and the aggregated power readings as the observed symbol. 


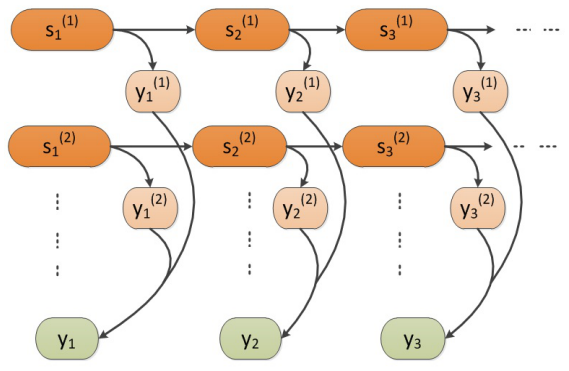

Fig. 2. Graphical representation of Factorial Hidden Markov Model.

\section{Estimation of Parameters and Hidden States}

The parameters for the HMM model are estimated using Expectation Maximization (EM) algorithm, which iteratively estimates the parameters value based on a auxiliary function until convergence to a local maximum occurs. The parameters estimated using EM are the number of chains, the number of states in each chain, the transition matrix of moving from one state to another and the initial state probability distribution. The goal of energy disaggregation is to identify accurately the states of the appliances in the aggregated signal. To identify the hidden states, the joint probability of the entire sequence of hidden states that generated a particular sequence of observations is calculated. We use Viterbi algorithm to estimate the hidden states of the HMMs. Viterbi algorithm uses dynamic programming to identify the most likely sequence of hidden states by finding a maximum over all possible state sequences.

\section{Our Approach For Sustainable Monitoring}

Contrary to prior work, we employ a semi-intrusive technique for identifying the minimal subset of appliances that needs to be individually monitored, in order to achieve high disaggregation accuracy. We propose an algorithm to model the total power consumption of the house accurately, as follows:

Step-1: We train the FHMM model using the aggregated signal (obtained using a single smart meter) and derive the possible number of chains, their states and transition matrix using EM clustering. Each chain derived is assumed to correspond to an appliance.

Step-2: We select a subset of appliances to be monitored individually (using plug-level meters), in order to improve the accuracy of disaggregation. Appliances monitored individually are modeled using HMMs to derive the number of states, transition matrix and initial state probabilities.

Step-3: We employ the derived HMM chains of individual appliances to the FHMM model and we retrain it using the aggregate power consumption. Thus, new chains may be added or existing ones modified. If the disaggregation accuracy is not high enough, then we go to Step-2.

There can be different approaches to select the subset of appliances that are individually monitored at Step-2. Here, we first describe a naive exhaustive search to identify the optimal subset by exploring all different combinations of appliances that belong to a house and select the one that provides the highest disaggregation accuracy (Subsection V-A). Then, we build upon this technique to derive a heuristic algorithm to identify the subset of appliances in a smarter way (Subsection V-B). Fig. 3 shows the complete model used to identify the usage of each appliances from the aggregated signal.

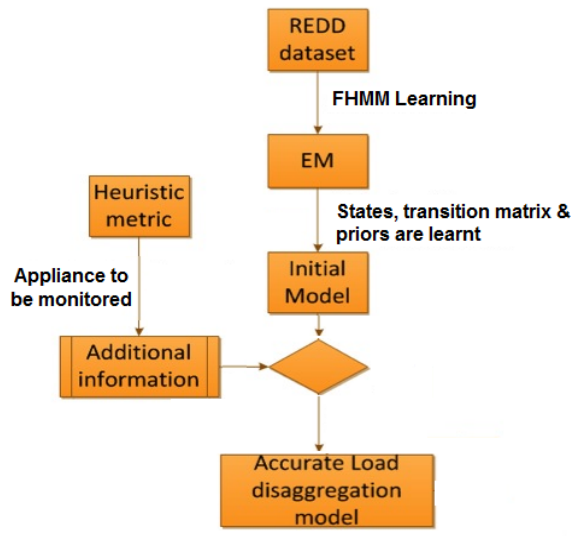

Fig. 3. Proposed Methodology using FHMM.

\section{A. Exhaustive search to find appliance subset}

A plug-level meter is employed to train the HMM model for an appliance. The trained model is employed in Step3 of the algorithm described above to update the FHMM model and the achievable disaggregation accuracy is measured. This is repeated for all combinations of appliances and the optimal subset of appliances that maximizes the energy disaggregation accuracy is found. There are several drawbacks of this approach: (a) All appliance plug-level monitoring data is required and thus plug-level meters need to be installed for all appliances in the house. (b) The installation and removal cost of plug-level meters are tedious and expensive. (c) The search time is exponential to the number of appliances in the house. This motivates us to develop a novel, smarter heuristic algorithm for identifying the optimal subset of appliances that maximizes the energy disaggregation accuracy.

\section{B. Heuristic to find subset of appliance to be monitored}

Let $C_{1}, C_{2}, \ldots, C_{n}$ be the set of chains derived from the FHMM model in Step-1 of Section V and let $\left(w_{1}, a_{1}\right),\left(w_{2}, a_{2}\right), \ldots,\left(w_{n}, a_{n}\right)$ be the pairs of weight (i.e. fraction of aggregate power consumption from an appliance) and individual accuracy for each chain derived. The goal of the heuristic, at each step, is to identify the pair $\left(w_{i}, a_{i}\right)$ that maximally improves the disaggregation accuracy $\alpha$, i.e.

$$
\begin{aligned}
& \alpha=w_{1} \cdot a_{1}+w_{2} \cdot a_{2}+\ldots+w_{n} \cdot a_{n} \\
& \arg _{i} \max w_{i} \cdot a_{i}
\end{aligned}
$$

It is intuitive to see that the appliance, which needs to be monitored, should be the one that has the highest power consumption; this appliance is expected to improve the overall disaggregation accuracy. In case of conflict in the weights, we select the pair which has the lowest $a_{i}$ value, as this is 
the chain that can improve the accuracy of disaggregation, if monitored. In our heuristic, we assume to know the number of appliances at each house and their states, their average and peak power consumption, as shown in Table I. We believe that this is trivial prior knowledge, which can be retrieved by the house residents by a questionnaire and by the appliance manuals. Algorithm 1 describes the procedure

TABLE I

AVERAGE, PEAK POWER CONSUMPTION \& NUMBER OF STATES FOR EACH APPLIANCE

\begin{tabular}{|c|c|c|c|}
\hline Appliance & $\begin{array}{c}\text { Average (\%) } \\
\text { consumption }\end{array}$ & $\begin{array}{c}\text { Peak (\%) } \\
\text { consumption }\end{array}$ & $\begin{array}{c}\text { Number of } \\
\text { states }\end{array}$ \\
\hline Refrigerator & $200 \mathrm{kWh}$ & $400 \mathrm{~W}$ & 3 \\
\hline Microwave & $60 \mathrm{kWh}$ & $1400 \mathrm{~W}$ & 3 \\
\hline Dish washer & $280 \mathrm{kWh}$ & $1000 \mathrm{~W}$ & 4 \\
\hline Washer Dryer & $512 \mathrm{kWh}$ & $2500 \mathrm{~W}$ & 4 \\
\hline
\end{tabular}

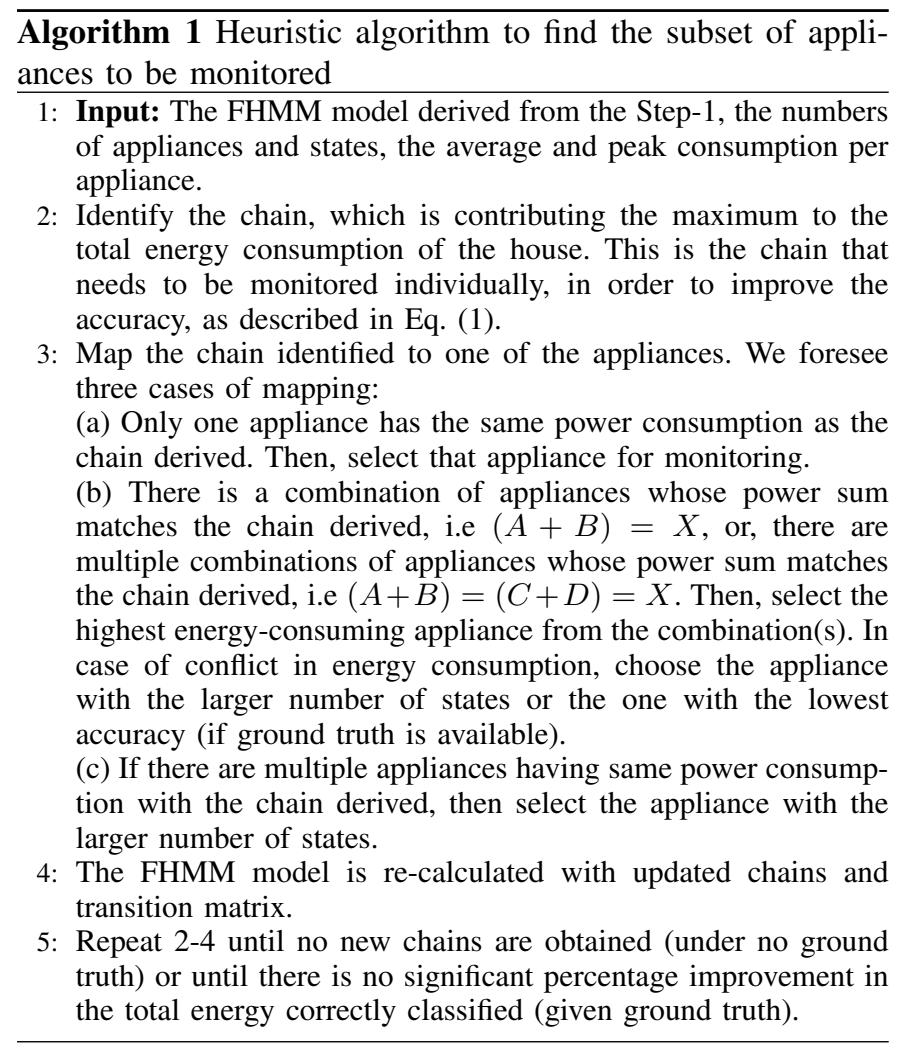

to find the subset of appliances. The heuristic considers the highest energy-consuming chain and tries to match it with one of the appliances from Table I using the steps (a), (b), (c), as described in Algorithm 1. Mapping the chain to an appliance is the core of the algorithm. There, we define three cases: (a) When there is an appliance that consumes the same average power with the chain derived, then we select that appliance for monitoring. In (b), we explore the possibility where the aggregate power consumption of one or multiple combinations of appliances matches to the highest power-consuming chain. In case that one appliance set matches the chain, we select the appliance from the set(s) that consumes the higher energy according to Table I. If multiple appliances consume the same energy, then the one with the more states should be selected, because of its higher complexity to be accurately estimated by the FHMM. The same way for appliance selection is employed in case (c), where multiple appliances have the same power consumption as the highest power-consuming chain. Once, the appliance to be monitored is selected, we use HMMs to model this appliance and derive the states, transition matrix and initial state distributions. We utilize this information to update the chains obtained from Step-1 and re-iterate the FHMM model to identify new chains.

This process is iterated until the number of chains equals the number of appliances or no new chains are found. This ensures that we select the minimum number of sensors to monitor, so that additional plug-level meters do not offer any drastic improvement in disaggregation accuracy. When historical data on each appliance power consumption is available (referred to as ground truth), then we can stop finding appliances when there is not significant improvement in disaggregation accuracy based on the ground truth. Using our heuristic, one can achieve very high disaggregation accuracy by installing only a small number of plug-meters for the appliances to be individually monitored. Note that, we do not consider any prior information in the initial step of modeling the FHMM. However, during the mapping of the appliances to chains we do use the information in Table I.

\section{EXPERIMENTAL EVALUATION}

In this section, we experimentally evaluate the proposed semi-intrusive power-consumption disaggregation approach and our heuristic based on a real dataset. The implementation has been done in Matlab. Below, we discuss the dataset employed, the performance metrics and present our results.

\section{A. Dataset}

To evaluate our proposed methodology, we use the publicly available Reference Energy Disaggregation Dataset (REDD) [6]. This dataset consists of power consumption information of six houses. For each house both aggregate and appliance level power readings were measured. Appliance-level consumption data were collected every 3 seconds using off-theshelf wireless plug monitor by Enmetric, while the houselevel consumption data was sampled every second using the eMonitor, developed by Powerhouse Dynamics. Both the aggregate and the appliance-level data were down sampled to one measurement every 20 seconds using a median filter. The dataset contains continuous data for at most 2 weeks per house.

\section{B. Evaluation Metrics}

We employ several accuracy metrics for the proposed energy disaggregation methodology. For the accuracy of the proposed approach, we use the ratio of the amount of energy that is correctly classified to the total energy consumed by the house. Also, we use precision, recall and F-measure to evaluate the classification accuracy of the energy usage per appliance. Given some energy estimated by the model to have been consumed by an appliance, precision refers to the amount of 
the assigned energy that was truly consumed by that appliance. Recall indicates the fraction of a given appliance energy that is correctly classified. F-measure is a combined measure of test accuracy and is defined as follows:

$$
F-\text { measure }=\frac{2 . \text { Precision } . \text { Recall }}{\text { Precision }+ \text { Recall }}
$$

We believe that evaluating based on the event detections may not be an accurate metric as, for most of the appliances, $\mathrm{OFF}$ instances are more prominent than $\mathrm{ON}$ ones. Hence, high accuracy based on the event detections may not indicate accurate classification of the $\mathrm{ON}$ instances, as opposed to our employed metrics.

\section{Modeling aggregated power using FHMM}

We discuss the results obtained from Step-1 of our proposed methodology, where we consider the aggregated power signal of a house and use EM clustering mechanism to derive the possible number of chains and their states, transition matrix and initial state probabilities. We use 2 weeks of continuous days data of house 1 of the REDD dataset as our training set and evaluate our technique on 4 random days of the month for the same house. We trained the FHMM on the training dataset and derived 4 chains with their corresponding mean power values at each state and transition matrix. Only the refrigerator appliance average power consumption was matching to one of the chain derived; the FHMM model was not able to correctly distinguish other appliances. We used the Viterbi algorithm to determine the possible set of states traversed for the test data. Table II shows the comparison of real energy consumed for each appliance and the estimated energy obtained from the FHMM model. From the table, we can infer that only $84 \%$ of energy estimated was correctly classified, however the model was not able to distinguish between set of appliances due to their same power consumption or their multiple states. Fig. 4 shows the real and estimated power using this approach.

TABLE II

REAL AND ESTIMATED POWER OBTAINED USING FHMM MODEL

\begin{tabular}{|c|c|c|}
\hline Appliance & Real power consumed (\%) & Estimated power (\%) \\
\hline Refrigerator & 25 & 34 \\
\hline GFI & 3 & 11 \\
\hline Microwave & 6 & \\
\hline Washer Dryer & 2 & 10 \\
\hline Dish washer & 3 & \\
\hline Lighting & 40 & \multirow{2}{*}{45} \\
\hline Kitchen outlets & 20 & \\
\hline
\end{tabular}

\section{Heuristic and Exhaustive Search Based Results}

As mentioned previously, the exhaustive search considers consumption data of each and every appliance in the house and iteratively finds out the best set that maximizes the energy disaggregation accuracy. Table III shows the improvement in energy classified correctly as number of appliances monitored increases. As seen in the table III, the percentage of energy correctly classified when one, two, three and four appliances were monitored in the house are $89.5 \%, 92.5 \%, 95 \%$ and $95.6 \%$ respectively. Fig. 5, shows the percentage of

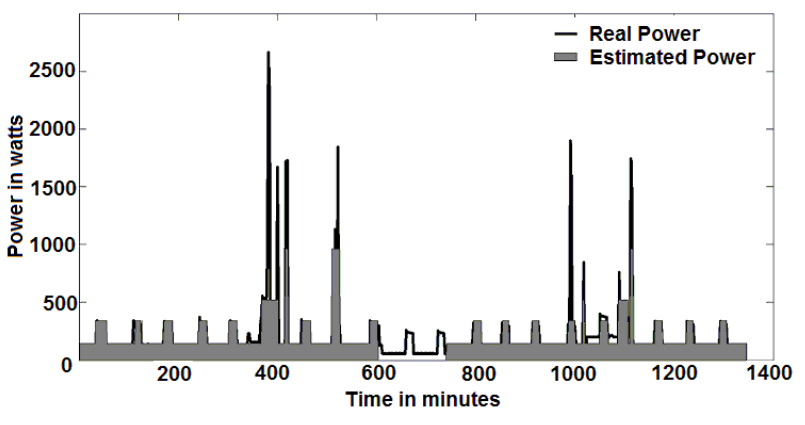

Fig. 4. Real and estimated power usage when no appliance is monitored.

energy correctly classified and the number of appliances that were identified. It can be clearly seen, as the number of appliances monitored increases, appliances identified in the house also increases. However, the total energy correctly classified and number of appliances identified does not have a high improvement after monitoring a certain number of appliances. This number is the minimum set of appliances that have to be monitored, in order to maximize the total energy correctly classified. We use this insight obtained (i.e. accuracy of the model does not increase beyond a threshold by adding more plug-level meters) in our heuristic as a stopping condition under no ground truth. We then use the heuristic

TABLE III

ENERGY DISAGGREGATION ACHIEVED BY MONITORING MULTIPLE APPLIANCES USING EXHAUSTIVE SEARCH

\begin{tabular}{|c|c|c|c|c|c|}
\hline Monitored Appliances & None & One & Two & Three & Four \\
\hline Refrigerator & 34 & 30.4 & 29.7 & 27.5 & 26.9 \\
\hline GFI & 11 & 13.8 & 3.3 & 4 & 3.5 \\
\cline { 5 - 6 } Microwave & & & 8.8 & 5.5 & 6 \\
\hline Washer Dryer & 10 & 5.7 & 5.7 & 3 & 2 \\
\hline Dish washer & & & & 3.5 & 3 \\
\hline Lighting & 45 & 50.1 & 52.5 & 30.5 & 33.6 \\
\cline { 1 - 3 } \cline { 5 - 6 } $\begin{array}{c}\text { Kitchen outlets } \\
\text { Energy correctly } \\
\text { classified }\end{array}$ & 84 & 89.5 & 92.5 & 95 & 95.6 \\
\hline
\end{tabular}

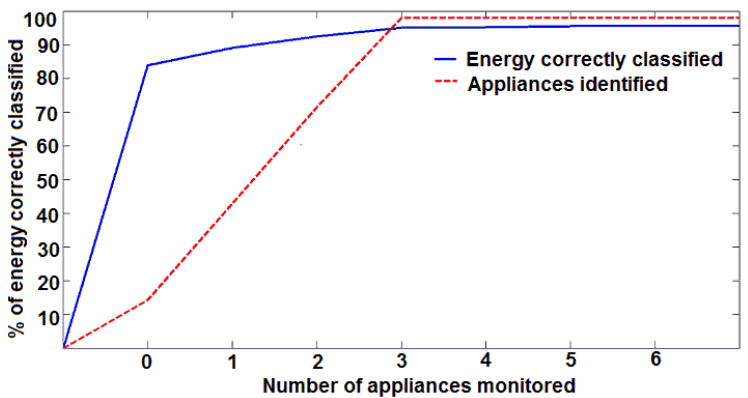

Fig. 5. Accuracy and number of appliances identified by the model.

metric described in Subsection V-B to identify the appliances to be individually monitored in a house. We apply our heuristic on the four chains of the FHMM derived from the test data when no appliance is monitored. We first identify Lighting and Kitchen outlets chain as having the highest energy consumption and hence we select this chain to be modeled 
TABLE IV

PRECISION, RECALL AND F-MEASURE PER IDENTIFIED APPLIANCE

\begin{tabular}{|c|c|c|c|}
\hline Appliance & Precision (\%) & Recall (\%) & F-measure \\
\hline Refrigerator & 92 & 85.2 & 0.88 \\
\hline GFI & 75 & 60 & 0.66 \\
\hline Microwave & 91.6 & 78.5 & 0.84 \\
\hline Washer Dryer & 83.3 & 66 & 0.73 \\
\hline Dish washer & 89.8 & 85.7 & 0.87 \\
\hline Lighting & 85 & 80 & 0.82 \\
\hline Kitchen outlets & 86 & 86.9 & 0.86 \\
\hline
\end{tabular}

individually. As this chain is a combination of appliances, we select the appliance in the combination that is the most energy-consuming one, i.e. Lighting (case (b) in Algorithm 1). The appliance selected is monitored individually and is modeled using HMM. Then, the appliance selection process is re-iterated with the updated FHMM. In the next iteration, the highest energy consuming chain identified is Refrigerator and hence we monitor and model it using HMM. The FHMM model is updated and the selection process is re-iterated until one of the stopping conditions in the heuristic algorithm is met. Finally, we derive \{Lighting, Refrigerator, Microwave\} as the subset of appliances to be individually monitored for house 1 of the REDD dataset. When these appliance set is individually monitored, the precision, recall and F-measure for each appliance achieved by the energy disaggregation process are shown in Table IV. Also, the total amount of energy correctly classified is $95 \%$. Fig. 6 shows the real and estimated power consumption on the test data. As compared to Fig. 4, using the subset of appliances derived from our heuristic, the system was able to identify all the appliances and correctly classify the energy. Any other subset of appliances would result in less disaggregation accuracy, as this is the same subset of appliances that the exhaustive search also obtained! Agreement between the exhaustive search and the heuristic was experimentally found for all houses of the REDD dataset. Thus, our heuristic saves computational, installation and maintenance costs, while performing similarly to the exhaustive search. Note that the set of appliances derived above is housespecific and depend on the appliances in the house and the consumption profiles of the residents. For instance, the sets of appliances \{Washer Dryer, Refrigerator\} and \{Bathroom GFI, Microwave, Refrigerator $\}$ were found to be monitored in house 2 and 3 of the REDD dataset respectively, in order to accurately disaggregate energy. It might also be the case that the set of appliances monitored in a house need to change for different seasons (e.g. winter, summer). We could not validate this claim due to lack of data.

\section{CONCLUSiOnS AND Future WORK}

We proposed a semi-intrusive approach for maximizing the accuracy of energy disaggregation per appliance in a sustainable way, i.e. using the minimum number of pluglevel meters that maximally improves disaggregation accuracy. Our approach employs FHMM and it was experimentally proved to achieve very high (i.e. 95\%) energy classification per appliance, while all appliances were discovered. Moreover,

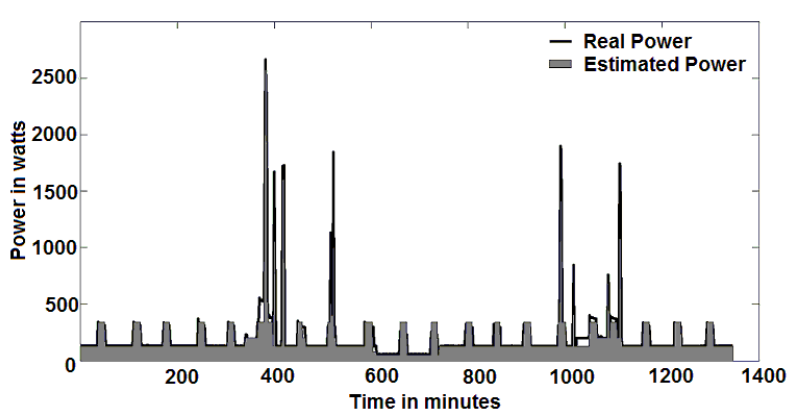

Fig. 6. Real and estimated power usage when 3 appliances are monitored.

we proposed a heuristic algorithm for the optimal selection of appliances to be individually monitored in a practical setting that saves computational, investment and maintenance costs. We believe that our approach is generic and can be employed for any residential setting to accurately disaggregate per appliance energy consumption in the most economic and least-intrusive way. As a future work, we plan to extend our approach for considering the installation of additional sensors (e.g. temperature, motion, etc.) apart from plug-level meters, in order to maximize energy disaggregation accuracy in a sustainable way.

\section{REFERENCES}

[1] M. Zeifman and K. Roth. Nonintrusive Appliance Load Monitoring (NIALM) for Energy Control in Residential Buildings: Review and Outlook. IEEE Transactions on Consumer Electronics, vol. 57, 2011.

[2] G. Hart. NonIntrusive Appliance Load Monitoring. In Proc. of the IEEE, 80(2):1870-1891, 1992.

[3] J. Paradiso, P. Dutta, H. Gellersen, E. Schooler. Smart Energy Systems. In Proc. of IEEE Pervasive Computing, 2011.

[4] A.G. Ruzzelli, C. Nicolas, A. Schoofs, G.M.P. O'Hare. Real-Time Recognition and Profiling of Appliances through a Single Electricity Sensor. In Proc. of SECON, 2010.

[5] S. Patel, T. Robertson, J. Kientz, M. Reynolds, G. Abowd. At the flick of a switch: detecting and classifying unique electrical events on the residential power line. In Proc. of UbiComp, 2007.

[6] J. Zico Kolter and Matthew J. Johnson. REDD: A public data set for energy disaggregation research. In Proc. of the SustKDD Workshop on Data Mining Applications in Sustainability, 2011.

[7] J. Z. Kolter, S. Batra, A. Y. Ng. Energy Disaggregation via Discriminative Sparse Coding. In Proc. of Neural Information Processing Systems, 2010.

[8] L. Farinaccio, R. Zmeureanu. Using a pattern recognition approach to disaggregate the total electricity consumption in a house into the major end-uses. Energy and Buildings, 30:245-259, 1999.

[9] Y. Kim, T. Schmid, Z. M. Charbiwala, M. B. Srivastava. ViridiScope: design and implementation of a fine grained power monitoring system for homes. In Proc. of UbiComp, 2009.

[10] T. Saitoh et al. Current Sensor based Non-intrusive Appliance Recognition for Intelligent Outlet In Proc. of the Int. Tech Conf on Circuits/Systems, Computers and Communications, 2008.

[11] O. Parson , S. Ghosh, M. Weal, A. Rogers Non-intrusive Load Monitoring using Prior Models of General Appliance Types. In Proc. of $A A A I, 2012$.

[12] Kim, H., Marwah, M., Arlitt, M.F., Lyon, G., Han, J. Unsupervised Disaggregation of Low Frequency Power Measurements. In Proc. of SIAM International Conference on Data Mining, 2011.

[13] O. Ardakanian, S. Keshav, and C. Rosenberg. Markovian models for home electricity consumption. In Proc. of the ACM SIGCOMM Workshop on Green Networking (GreenNets '11), 2011.

[14] T. Kato, H. S. Cho, D. Lee, T. Toyomura and T. Yamazaki. Appliance Recognition from Electric Current Signals for Information-Energy Integrated Network in Home Environments. In Proc. of ICOST, 2009.

[15] G. Zoubin, J. Michael. Factorial Hidden Markov Models. Machine Learning, 29(2-3):245-273, 1997. 